\title{
EFEITO DO EXTRATO HIDROALCÓOLICO DE Polygonum hydropiperoides EM CÉLULAS ESPERMÁTICAS DE SUs scrofa domesticus
}

\author{
GIORDANI, Claudia ${ }^{1}$; \\ SILVA, Cristine Cioato da ${ }^{1}$; \\ PERERA, Soliane Carra ${ }^{2}$; \\ BARBOSA, Rafael Mielke ${ }^{2}$; \\ PIRES, Diego Martins ${ }^{3}$; \\ CORCINI, Carine Dahl ${ }^{3}$; \\ VARELA JÚNIOR, Antônio Sérgio ${ }^{4}$; \\ CLEFF, Marlete Brum ${ }^{5}$.
}

\footnotetext{
${ }^{1}$ Programa de Pós-Graduação em Veterinária, Faculdade de Veterinária, Universidade Federal de Pelotas, ${ }^{2}$ Faculdade de Veterinária, Universidade Federal de Pelotas; ${ }^{3}$ Departamento de Patologia Animal, Faculdade de Veterinária, Universidade Federal de Pelotas; ${ }^{4}$ Instituto de Ciências Biológicas, Universidade Federal do Rio Grande; ${ }^{5}$ Departamento de Clínicas Veterinárias, Faculdade de Veterinária, Universidade Federal de Pelotas.
}

\section{RESUMO}

$P$ olygonum hydropiperoides é uma das plantas utilizadas como medicinal e que apresenta atividades biológicas confirmadas na dependência da composição química. Desta forma, estudos sobre a toxicidade e interação desta planta com as células são necessários, sendo os espermatozoides uma opção inovadora para a avaliação. Assim, o objetivo deste estudo foi determinar a composição química do extrato hidroalcoólico de $P$. hydropiperoides (PH) e a toxicidade em células espermáticas. Para a determinação da composição química do extrato foi utilizada a análise cromatográfica líquida de ultra eficiência. A toxicidade foi avaliada através da análise computadorizada da motilidade espermática de sêmen suíno doméstico (Sus scrofa domesticus), enquanto as alterações celulares foram analisadas por citometria de fluxo. Foram identificados no extrato vegetal um total de 17 compostos fenólicos destacando-se o ácido quínico e quercetina. Os parâmetros avaliados não sofreram alterações significativas nas concentrações de 1,5 até 6,2 $\mathrm{mg} \cdot \mathrm{mL}^{-1}$ independente do tempo de exposição ao extrato, sendo que a redução dos parâmetros ocorreu de forma progressiva ao aumento das concentrações $\left(6,2 \mathrm{mg} \cdot \mathrm{mL}^{-1} \leq 12\right.$ $\left.\mathrm{mg} \cdot \mathrm{mL}^{-1} \leq 50 \mathrm{mg} \cdot \mathrm{mL}^{-1} \leq 100 \mathrm{mg} \cdot \mathrm{mL}^{-1}\right)$. Através da citometria de fluxo pode-se observar que em nível de estrutura celular, não foram observadas alterações significativas. As alterações provocadas pelo extrato podem sugerir os possíveis mecanismos de ação a serem explorados para aplicação clínica. $O$ extrato hidroalcoólico de $P$. hydropiperoides, abundante em ácido quínico e quercetina, acarretou apenas em alterações na motilidade espermática, a partir de $12 \mathrm{mg} \cdot \mathrm{mL}^{-1}$.

Palavras-chave: Toxicidade. Plantas. Motilidade espermática. Citometria de fluxo. CASA. 


\section{INTRODUÇÃO}

Produtos provenientes de plantas podem ser tóxicos, mesmo quando obtidos de espécies utilizadas popularmente como medicinais, sendo necessários estudos para elucidação da composição, qualidade, eficácia e segurança destes recursos (ZÖLLNER; SCHWARZ, 2013). A pesquisa científica com espécies vegetais tem sido incentivada e intensificada devido a importância da utilização das plantas medicinais e a possibilidade de obtenção de compostos bioativos para tratamento de enfermidades e combate de microrganismos resistentes (BARBOSA-FILHO et al., 2007; CECHINEL FILHO; YUNES, 1998). A Organização Mundial de Saúde (OMS) considera as plantas medicinais como a melhor fonte para o desenvolvimento de uma variedade de fármacos (PRASHAR et al., 2003). Dada sua importância no Brasil, o Ministério da Saúde criou o Programa Nacional de Plantas Medicinais e Fitoterápicos e a Relação Nacional de Plantas Medicinais de Interesse ao Sistema Único de Saúde (RENISUS). Os objetivos desses programas são de ampliar ações terapêuticas e melhorias à atenção básica no Sistema Único de Saúde (SUS), incentivar o uso sustentável da biodiversidade nacional, valorizar e preservar o conhecimento tradicional, além da listagem das plantas com potencial para geração de produtos e da orientação às pesquisas para elaboração de fitoterápicos eficazes e seguros (BRASIL, 2009a, 2009b).

Dentre as plantas citadas na lista do RENISUS está a Polygonum hydropiperoides Michaux (Polygonaceae, erva-de-bicho), considerada como planta invasora, tendo uso popular no tratamento de feridas, além de ação anti-inflamatória, antiparasitária, antidiarreica, antihemorroidal, adstringente e diurética (BRANDÃO et al., 2012; BUSSMANN et al., 2010; LORENZI; MATOS, 2008). Estudos científicos são escassos, mas demonstram atividade antifúngica e antibacteriana (BUSSMANN et al., 2008; FREIXA et al., 1998).

Apesar da indicação popular e da comprovação científica da atividade, pesquisas que avaliem toxicidade e o local de ação de extratos vegetais, especialmente para $P$. hydropiperoides, ainda são incipientes (BIANCHI et al., 1993; CUNHA et al., 2016). Técnicas que utilizem células como indicador, devem ser consideradas inicialmente, de modo a viabilizar a avaliação da toxicidade dos extratos, a determinação dos mecanismos de ação, reduzindo, assim, o uso de animais de laboratório. Neste contexto, se destacam as células 
espermáticas, por possuírem semelhança com células somáticas e demonstrarem grande sensibilidade a toxinas (ANDERSSON et al., 2006, 2010; HOORNSTRA et al., 2003; JÄÄSKELÄINEN et al., 2003; SEVERIN et al., 2005). Como vantagem, também pode ser destacada a facilidade de obtenção de dados e avaliação através de técnicas computadorizadas/automatizadas como o CASA (Computer-Assited Sperm Analysis) e a citometria de fluxo, na qual é possível verificar alterações de parâmetros espermáticos. 0 objetivo deste estudo foi determinar a composição química e a citotoxicidade, em diferentes tempos de exposição, do extrato hidroalcoólico de $P$. hydropiperoides em células espermáticas.

\section{MATERIAL E MÉTODOS}

O material vegetal foi obtido (Pelotas-RS, $31^{\circ} 33^{\prime} 02.0^{\prime \prime} \mathrm{S}, 52^{\circ} 16^{\prime} 45.5^{\prime \prime} \mathrm{W}$ ) e depositado no Herbário do Departamento de botânica da Universidade Federal de Pelotas, sendo registrado sob o número 25931 - P. hydropiperoides Michaux (erva-de-bicho). Para preparação do extrato hidroalcoólico, misturou-se $100 \mathrm{~g}$ de folhas secas trituradas com 500 $\mathrm{mL}$ de álcool de cereais a $70 \%$. Após, foi realizada a rotaevaporação à vácuo com banho de aquecimento $\left(50{ }^{\circ} \mathrm{C}\right)$, sendo o volume inicial restituído com água destilada estéril (SCHIEDECK et al., 2008).

Para avaliar a composição química dos extratos foram injetados (cromatógrafo líquido de ultra-alta eficiência - Shimadzu, Prominence, acoplado a espectrômetro de massas de alta resolução - Impact HD) $10 \mu \mathrm{L}$ da amostra (300 $\left.\mu \mathrm{g} \cdot \mathrm{mL}^{-1}\right)$ diluída em metanol. A separação dos compostos fenólicos ocorreu através da utilização de pré-coluna C18 $(2,0 \times 4 \mathrm{~mm})$ e coluna Luna C18 (2,0 x $150 \mathrm{~mm}, 100 \AA$, $3 \mu \mathrm{m})$ Phenomenex (Torrance, CA, USA). Os compostos fenólicos foram identificados através da comparação do tempo de retenção com os padrões externos, espectro de massa e perfil de fragmentação.

Para as avaliações de toxicidade celular, foram coletados três ejaculados $(n=3)$ de cada macho $(n=3)$ e cada amostra foi processada em triplicata, fazendo a utilização da fração rica do sêmen dos suínos domésticos (Sus scrofa domesticus), com motilidade espermática $>70 \%$, diluída (1:1) em Beltsville Thawing Solution (BTS). Para os tratamentos, foi adicionado ao sêmen o extrato hidroalcoólico de $P$. hydropiperoides (previamente aferindo o $\mathrm{pH}$ da 
solução de estoque), diluídos em BTS até obtenção das concentrações finais de 0 (controle negativo: apenas BTS); 1,5; 6,2; 12,5; 50 e $100 \mathrm{mg}^{-\mathrm{mL}^{-1}}$. O efeito do extrato foi observado nos tempos 0, 24 e 48 horas de contato com o sêmen, armazenados sob refrigeração $\left(17^{\circ} \mathrm{C}\right)$. A cinética espermática foi avaliada através da análise computadorizada de espermatozoides (CASA) no equipamento Axio Scope A1 Ax10 (Zeiss), com o programa Sperm Vision @), em condições isotérmicas de $37^{\circ} \mathrm{C}$, sendo realizada a média de leitura em 10 campos $(\geq 1000$ células/campo). Os parâmetros avaliados foram motilidade total (MT-\%), motilidade progressiva (MP-\%), distância média percorrida (DAP-mm), distância curvilínea (DCL), distância linear (DSL-mm), velocidade média da trajetória (VAP), velocidade curvilínea (VCL$\mathrm{mm} / \mathrm{s}$ ), velocidade linear progressiva (VSL-mm/s), frequência do batimento flagelar cruzado (BCF-Hz), amplitude do deslocamento lateral da cabeça (ALH-mm), oscilação (WOB-Hz), linearidade (LIN-\%) e retilinearidade (STR-\%).

Os parâmetros acrossoma não reagido (ANR), integridade de membrana (IM), fluidez da membrana (F), potencial de membrana mitocondrial (PMM), índice de fragmentação de DNA (DFI), espécies reativas de oxigênio (ROS) e peroxidação lipídica (LPO) foram avaliados por citometria de fluxo (Attune ${ }^{\circledR}$ Acoustic Focusing - Life Tecnologies) no dia 0 (toxicidade aguda), também utilizando a fração rica de três ejaculados $(n=3)$ dos mesmos animais $(n=3)$, em triplicata. Foi utilizado laser violeta $(\lambda=405 \mathrm{~nm})$ e as células foram coradas com Hoechst 33342, exceto para a avaliação de DNA (concentração de $16,2 \mathrm{mM}$ ), sendo eliminados efeitos não-espermáticos (MARTINEZ-ALBORCIA et al., 2012). Para as leituras foram adicionados $2 \mathrm{~mL}$ de tampão fosfato salino (PBS) sem cálcio (80 g NaCl, 11,5 g KCl, $24 \mathrm{~g}$ $\mathrm{Na}_{2} \mathrm{HPO}_{4}, 2 \mathrm{~g} \mathrm{KH}_{2} \mathrm{PO}_{4}$ e $1 \mathrm{~L}$ água Mili-Q), apresentando 20.000 eventos/análise com fluxo de 200 células. Foram utilizadas sondas fluorescentes específicas para cada parâmetro avaliado, permanecendo a amostra por cinco minutos, em ausência de luz a $37^{\circ} \mathrm{C}$ e após adicionado $500 \mu \mathrm{l}$ de PBS sem cálcio.

$\mathrm{Na}$ avaliação de acrossoma, foram utilizados os corantes de lectina de amendoim (PNA) associado ao isotiocianato de fluoresceína (FITC) $\left(1 \mu \mathrm{g} \cdot \mathrm{ml}^{-1}\right)$ (FITC-PNA), e o iodeto de propídio (IP) (7,3 $\mu \mathrm{M})$. Os resultados foram expressos em \% de células com o acrossoma não reagido (FITC-PNA - e a IP -) (MARTINEZ-PASTOR et al., 2010). 
A integridade de membrana espermática foi avaliada através da inserção dos corantes 27 $\mathrm{mM}$ carboxifluoresceína diacetato (DFC) e 7,5 mM de IP, sendo os resultados expressos em \% de células não danificadas (DFC+IP-) (FERNANDEZ-GAGO et al., 2013).

A avaliação do DNA foi realizada com o corante acridina laranja, em $10 \mu \mathrm{L}$ de sêmen foi adicionado $5 \mu \mathrm{L}$ de tampão TNE (0,01 M Tris- $\mathrm{HCl}, 0,15 \mathrm{M} \mathrm{NaCl}, 0,001 \mathrm{M}$ EDTA, pH 7,2) por 30 segundos, $10 \mu \mathrm{L}$ de Triton (0,1\% Triton X-100) por 30 segundos, e $5 \mu \mathrm{L}$ de acridina laranja (ácido cítrico 0,037 M, $\mathrm{Na}_{2} \mathrm{HPO}_{4}$ 0,126 M, 0,0011 M de EDTA dissódico, $\mathrm{NaCl}$ 0,15 M, pH 6,0) (EVENSON; JOST, 1994). As leituras foram realizadas em até cinco minutos, sendo os resultados expressos em índice de DNA fragmentado.

As espécies reativas de oxigênio (ROS) foram avaliadas com 2'7'diclorofluoresceína diacetato (H2DCF-DA) (1 uM) e 7,3 $\mu \mathrm{M}$ de IP, sendo os resultados expressos em intensidade da fluorescência verde de espermatozoides não danificados (DOMÍNGUEZ-REBOLLEDO et al., 2010).

A função mitocondrial foi avaliada através da sonda rodamina 123 (Rh123) (13 uM) e IP (7,3 uM). Os resultados foram expressos em $\%$ de espermatozoides intactos com alta funcionalidade mitocondrial (alta fluorescência e alto acúmulo Rh123) (SILVA et al., 2016).

A lipoperoxidação (LPO) foi medida utilizando 0,002 mM C11 BODIPY 581/591, sendo os resultados expressos através das fluorescências verde e vermelha após duas horas, considerando a \% de presença de LPO (SILVA et al., 2013).

Para a análise estatística foi utilizado o software Statistix $9.0^{\circledR}$, sendo dados com normalidade, foi utilizado o teste ANOVA e as médias foram comparadas através do teste de Tukey.

\section{RESULTADOS E DISCUSSÃO}

Através da cromatografia líquida, identificou-se 17 compostos fenólicos no extrato de $P$. hydropiperoides, sendo majoritários o ácido quínico e a quercetina (Tabela 1). 
Tabela 1 - Compostos químicos do extrato hidroalcoólico de Polygonum hydropiperoides (PH), identificados por cromatografia líquida de ultra-eficiência.

\begin{tabular}{|c|c|c|c|}
\hline $\mathbf{N}^{\circ}$ & RT (min) & Identificação & $\mathrm{PH}\left(\mathrm{mg} \cdot \mathrm{g}^{-1}\right)$ \\
\hline 1 & 2.83 & Ácido quínico & 3.83 \\
\hline 2 & 5.49 & Ácido gálico & 0.07 \\
\hline 3 & 10.28 & Catequina & 0.20 \\
\hline 4 & 11.27 & Ácido Clorogênico & 0.38 \\
\hline 5 & 12.05 & Ácido cafeico & 0.04 \\
\hline 6 & 13.24 & Etil galato & 0.24 \\
\hline 7 & 13.67 & Ácido cumárico & 0.02 \\
\hline 8 & 14.01 & Ácido Ferrúlico & 0.04 \\
\hline 9 & 14.41 & Miricitrina & 0.38 \\
\hline 10 & 15.08 & Quercetina 3-Arabinose ${ }^{2}$ & 1.34 \\
\hline 11 & 15.21 & Ácido Elágico & 0.07 \\
\hline 12 & 15.58 & Miricetina & 0.13 \\
\hline 13 & 16.89 & Quercetina & 4.12 \\
\hline 14 & 18.00 & Kaempferol $^{1}$ & 0.03 \\
\hline 15 & 19.41 & Pinocembrina $^{1}$ & 0.02 \\
\hline 16 & 19.62 & $\begin{array}{l}\text { 3',5-Di-hidroxi-3,4',5',7- } \\
\text { tetramethoxiflavona }^{2}\end{array}$ & 0.52 \\
\hline 17 & 20.12 & Crisina & 0.01 \\
\hline
\end{tabular}

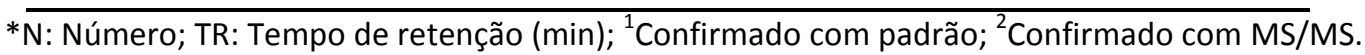

Na avaliação da motilidade espermática, após contato com o extrato de $P$. hydropiperoides $(\mathrm{SE}=\mathrm{pH} 4,59)$, observou-se no momento 0 horas, alterações somente a partir da concentração $12,5 \mathrm{mg} \cdot \mathrm{mL}^{-1}$, com redução somente da motilidade progressiva das células. A redução na motilidade total foi observada a partir da concentração $50 \mathrm{mg} \cdot \mathrm{mL}^{-1}$, em todos os tempos de exposição, porém com maior intensidade em $100 \mathrm{mg} \cdot \mathrm{mL}^{-1}$ (Figura 1). 


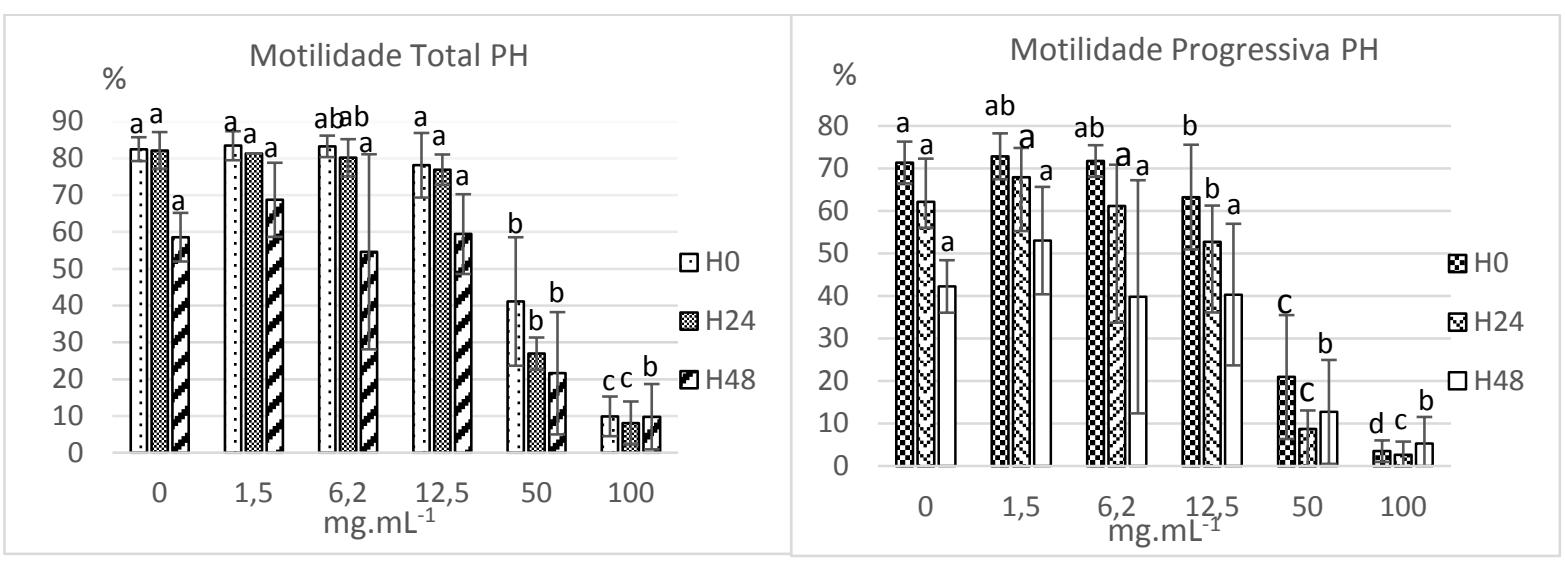

Figura 1 - Motilidade total e progressiva média (\%) ( \pm Desvio Padrão) de espermatozoides suínos frente a diferentes concentrações e tempos de exposição ao extrato de Polygonum hydropiperoides (PH). ${ }^{\text {abc }}$ Letras diferentes correspondem a diferença estatística entre os tratamentos no mesmo tempo de exposição $(p<0,05)$.

De modo geral, todos os parâmetros espermáticos avaliados não sofreram alterações significativas na concentração de $1,5 \mathrm{mg} \cdot \mathrm{mL}^{-1}$ independente do tempo de exposição, sendo que a redução dos parâmetros avaliados ocorreu de forma progressiva ao aumento das concentrações $\left(6,2 \mathrm{mg} \cdot \mathrm{mL}^{-1} \leq 12 \mathrm{mg} \cdot \mathrm{mL}^{-1} \leq 50 \mathrm{mg} \cdot \mathrm{mL}^{-1} \leq 100 \mathrm{mg} \cdot \mathrm{mL}^{-1}\right)$.

No momento 0 , houve redução de parâmetros nas concentrações 50 e $100 \mathrm{mg} \cdot \mathrm{mL}^{-1}$, exceto com relação a oscilação, linearidade e retilinearidade que não foram alterados. Em 24 horas, observou-se redução da distância média percorrida e linear (DAP e DSL) a partir de 6,2 $\mathrm{mg} \cdot \mathrm{mL}^{-1}$, enquanto que com $12,5 \mathrm{mg} \cdot \mathrm{mL}^{-1}$ observou-se alteração em mais quatro parâmetros de distância e velocidade (DAP, DSL, VSL, DCL, VAP e VCL), e em $50 \mathrm{mg} \cdot \mathrm{mL}^{-1}$ a frequência dos batimentos também foi alterada (DAP DSL, VSL, DCL, VAP, VCL e BCF), enquanto na concentração $100 \mathrm{mg} \cdot \mathrm{mL}^{-1}$ somente a oscilação (WOB) não sofreu alteração. Entretanto em 48 horas, as alterações iniciaram em $50 \mathrm{mg} \cdot \mathrm{mL}^{-1}$ (apenas em VAP) e em $100 \mathrm{mg} \cdot \mathrm{mL}^{-1}$ houve reduções em quase totalidade dos parâmetros espermáticos (Tabela 2). O STR, em todos os momentos e nas concentrações testadas, não apresentou mudanças significativas. 
Tabela 2 - Parâmetros de motilidade espermática (média desvio padrão) frente ao extrato hidroalcoólico de Polygonum hydropiperoides (PH) nos tempos de exposição 0, 24 e 48 horas.

\begin{tabular}{|c|c|c|c|c|c|c|}
\hline \multirow{2}{*}{ Parâmetros } & \multicolumn{6}{|c|}{ PH Concentração (mg.mL ${ }^{-1}$ ) 0 Horas } \\
\hline & 0 (BTS) & 1,5 & 6,2 & 12,5 & 50 & 100 \\
\hline $\operatorname{DAP}(\mu \mathrm{m} / \mathrm{s})$ & $27,20 \pm 3,48^{A}$ & $27,84 \pm 0,15^{\mathrm{A}}$ & $28,20 \pm 0,14^{\mathrm{A}}$ & $27,18 \pm 4,60^{A}$ & $18,96 \pm 2,64^{B}$ & $16,67 \pm 8,55^{\mathrm{B}}$ \\
\hline $\mathrm{DCL}(\mu \mathrm{m} / \mathrm{s})$ & $53,30 \pm 7,08^{A}$ & $53,88 \pm 3,65^{A}$ & $54,24 \pm 7,32^{A}$ & $51,84 \pm 8,87^{\mathrm{A}}$ & $36,61 \pm 5,82^{B}$ & $29,48 \pm 18,36^{B}$ \\
\hline $\mathrm{DSL}(\mu \mathrm{m} / \mathrm{s})$ & $19,44 \pm 2,02^{A}$ & $20,17 \pm 1,37^{A}$ & $20,44 \pm 2,64^{A}$ & $19,97 \pm 2,78^{A}$ & $13,87 \pm 2,03^{B}$ & $13,26 \pm 7,33^{B}$ \\
\hline $\operatorname{VAP}(\mu \mathrm{m} / \mathrm{s})$ & $59,89 \pm 8,42^{A}$ & $60,69 \pm 3,82^{A}$ & $61,95 \pm 7,27^{A}$ & $59,29 \pm 9.63^{A}$ & $41,34 \pm 4,58^{B}$ & $39,23 \pm 18,07^{B}$ \\
\hline $\operatorname{VCL}(\mu \mathrm{m} / \mathrm{s})$ & $116,86 \pm 16,87^{\mathrm{A}}$ & $117,58 \pm 7,57^{A}$ & $118,89 \pm 15,30^{A}$ & $112,79 \pm 18,46^{A}$ & $79,56 \pm 10,47^{B}$ & $68,09 \pm 38,59^{B}$ \\
\hline VSL $(\mu \mathrm{m} / \mathrm{s})$ & $43,03 \pm 4,99^{A}$ & $44,30 \pm 2,80^{A}$ & $45,07 \pm 5,47^{\mathrm{A}}$ & $43,61 \pm 5,72^{A}$ & $30,29 \pm 3,51^{B}$ & $31,00 \pm 15,48^{B}$ \\
\hline STR (\%) & $0,71 \pm 0,03^{A}$ & $0,72 \pm 0,01^{A}$ & $0,71 \pm 0,01^{A}$ & $0,73 \pm 0,03^{A}$ & $0,73 \pm 0,07^{A}$ & $0,72 \pm 0,23^{A}$ \\
\hline LIN (\%) & $0,36 \pm 0,02^{A}$ & $0,37 \pm 0,00^{A}$ & $0,37 \pm 0,01^{A}$ & $0,38 \pm 0,02^{A}$ & $0,38 \pm 0,05^{A}$ & $0,45 \pm 0,19^{A}$ \\
\hline WOB $(\mu \mathrm{m} / \mathrm{s})$ & $0,50 \pm 0,01^{A}$ & $0,51 \pm 0,00^{A}$ & $0,51 \pm 0,00^{A}$ & $0,52 \pm 0,01^{A}$ & $0,51 \pm 0,02^{A}$ & $0,57 \pm 0,20^{A}$ \\
\hline ALH $(\mu \mathrm{m} / \mathrm{s})$ & $2,86 \pm 0,35^{A}$ & $2,85 \pm 0,15^{A B}$ & $2,91 \pm 0,14^{\mathrm{A}}$ & $2,81 \pm 0,32^{A B}$ & $2,28 \pm 0,44^{\mathrm{BC}}$ & $2,21 \pm 1,07^{\mathrm{C}}$ \\
\hline $\mathrm{BCF}(\mathrm{Hz})$ & $39,27 \pm 0,35^{A}$ & $39,80 \pm 1,03^{A}$ & $39,25 \pm 1,84^{A}$ & $38,02 \pm 1,18^{\mathrm{AB}}$ & $32,52 \pm 3,72^{\mathrm{B}}$ & $31,81 \pm 12,96^{\mathrm{B}}$ \\
\hline \multicolumn{7}{|c|}{24 Horas } \\
\hline DAP & $21,83 \pm$ & $24,53 \pm 1,50^{A}$ & $20,76 \pm 1,46^{B}$ & $18,71 \pm 1,73^{\mathrm{B}}$ & $14,92 \pm 2,48^{C}$ & $6,54 \pm 5,96^{D}$ \\
\hline $\mathrm{DCL}(\mu \mathrm{m} / \mathrm{s})$ & $41,15 \pm 2,36^{A}$ & & $37,14 \pm 2,10^{\mathrm{AB}}$ & $32,83 \pm 3,32^{\mathrm{BC}}$ & $27,62 \pm 5,95^{\mathrm{C}}$ & $12,75 \pm 12,05^{D}$ \\
\hline $\mathrm{DSL}(\mu \mathrm{m} / \mathrm{s})$ & $16,64 \pm 1,46^{\mathrm{AB}}$ & $18,17 \pm 1,15^{A}$ & $15,00 \pm 1,03^{\mathrm{BC}}$ & $13,71 \pm 1,38^{C}$ & $10,90 \pm 2,59^{D}$ & $4,28 \pm 4,00^{\mathrm{E}}$ \\
\hline $\operatorname{VAP}(\mu \mathrm{m} / \mathrm{s})$ & $48,27 \pm 2,97^{\mathrm{A}}$ & $55,00 \pm 3,67^{A B}$ & $47,09 \pm 3,69^{\mathrm{AB}}$ & $42,80 \pm 4,34^{B}$ & $34,00 \pm 4,85^{c}$ & $16,64 \pm 15,30^{D}$ \\
\hline $\mathrm{VCL}(\mu \mathrm{m} / \mathrm{s})$ & $90,67 \pm 5,46^{A}$ & $97,06 \pm 5,24^{A}$ & $83,92 \pm 4,96^{\mathrm{AB}}$ & $74,75 \pm 8,47^{\mathrm{BC}}$ & $62,19 \pm 10,59^{C}$ & $32,58 \pm 31,19^{D}$ \\
\hline VSL $(\mu \mathrm{m} / \mathrm{s})$ & $36,79 \pm 3,24^{A}$ & $40,80 \pm 2,82^{\mathrm{AB}}$ & $34,05 \pm 2,54^{\mathrm{A}}$ & $31,33 \pm 2,94^{\mathrm{B}}$ & $24,98 \pm 4,97^{C}$ & $11,05 \pm 10,57^{D}$ \\
\hline STR (\%) & $0,75 \pm 0,03^{A}$ & $0,73 \pm 0,01^{A}$ & $0,71 \pm 0,01^{A}$ & $0,72 \pm 0,05^{A}$ & $0,72 \pm 0,07^{A}$ & $0,37 \pm 0,34^{B}$ \\
\hline LIN (\%) & $0,40 \pm 0,02^{A}$ & $0,41 \pm 0,01^{A}$ & $0,40 \pm 0,01^{A}$ & $0,41 \pm 0,04^{\mathrm{A}}$ & $0,40 \pm 0,07^{A}$ & $0,20 \pm 0,21^{\mathrm{B}}$ \\
\hline WOB $(\mu \mathrm{m} / \mathrm{s})$ & $0,52 \pm 0,01^{A}$ & $0,56 \pm 0,01^{A}$ & $0,55 \pm 0,02^{A}$ & $0,56 \pm 0,02^{A}$ & $0,55 \pm 0,08^{A}$ & $0,30 \pm 0,30^{A}$ \\
\hline ALH $(\mu \mathrm{m} / \mathrm{s})$ & $2,89 \pm 0,29^{A}$ & $3,24 \pm 0,15^{A}$ & $3,17 \pm 0,17^{A}$ & $2,98 \pm 0,36^{A}$ & $2,70 \pm 0,54^{A}$ & $1,61 \pm 1,50^{B}$ \\
\hline z) & $36,61 \pm 2,42^{A}$ & $35,36 \pm 1,42^{A}$ & $33,55 \pm 1,01^{\mathrm{AB}}$ & $32,17 \pm 2,26^{\mathrm{AB}}$ & $27,55 \pm 5,04^{B}$ & $14,34 \pm 13,83^{C}$ \\
\hline \multicolumn{7}{|c|}{48 Horas } \\
\hline DAI & $37 \pm 3,32^{A}$ & $24,22 \pm 1,37^{A}$ & $20,71 \pm 7,24^{\mathrm{A}}$ & $22,25 \pm 0,29^{A}$ & $18,66 \pm 4,06^{\mathrm{A}}$ & $9,31 \pm 9,73^{B}$ \\
\hline DCL & $44,50 \pm 5$ & $46,86 \pm 1,61^{A}$ & $41,02 \pm 14,84^{\mathrm{A}}$ & $43,09 \pm 6,81^{A}$ & $36,44 \pm 11,12^{\mathrm{A}}$ & $19,03 \pm 20,00^{\mathrm{B}}$ \\
\hline $\mathrm{DSL}(\mu \mathrm{m} / \mathrm{s})$ & $16,13 \pm 1,68^{A}$ & $18,60 \pm 1,22^{A}$ & $15,24 \pm 6,00^{A}$ & $17,32 \pm 2,97^{A}$ & $15,02 \pm 4,15^{\mathrm{A}}$ & $7,07 \pm 7,41^{\mathrm{B}}$ \\
\hline $\operatorname{VAP}(\mu \mathrm{m} / \mathrm{s})$ & $49,36 \pm 8,75^{\mathrm{AB}}$ & $53,11 \pm 3,37^{A}$ & $45,26 \pm 15,95^{A B}$ & $48,86 \pm 6,68^{\mathrm{AB}}$ & $39,67 \pm 7,85^{\mathrm{B}}$ & $19,89 \pm 20,86^{C}$ \\
\hline $\mathrm{VCL}(\mu \mathrm{m} / \mathrm{s})$ & $97,65 \pm 13,90^{\mathrm{A}}$ & $102,46 \pm 4,14^{\mathrm{A}}$ & $89,42 \pm 32,57^{A}$ & $94,14 \pm 14,45^{A}$ & $76,98 \pm 22,03^{A}$ & $40,74 \pm 42,97^{B}$ \\
\hline VSL $(\mu \mathrm{m} / \mathrm{s})$ & $35,50 \pm 3,93^{A}$ & $40,77 \pm 2,75^{A}$ & $33,35 \pm 13,18^{A}$ & $38,03 \pm 6,25^{A}$ & $31,92 \pm 8,23^{A}$ & $15,02 \pm 15,77^{B}$ \\
\hline STR (\%) & $0,72 \pm 0,08^{A}$ & $0,76 \pm 0,02^{A}$ & $0,67 \pm 0,21^{A}$ & $0,77 \pm 0,03^{A}$ & $0,79 \pm 0,05^{A}$ & $0,37 \pm 0,39^{A}$ \\
\hline LIN (\%) & $0,36 \pm 0,03^{A}$ & $0,39 \pm 0,02^{A}$ & $0,33 \pm 0,11^{A}$ & $0,39 \pm 0,02^{A}$ & $0,41 \pm 0,06^{A}$ & $0,18 \pm 0,19^{B}$ \\
\hline WOB $(\mu \mathrm{m} / \mathrm{s})$ & $0,49 \pm 0,02^{A}$ & $0,51 \pm 0,02^{A}$ & $0,46 \pm 0,13^{A}$ & $0,51 \pm 0,02^{A}$ & $0,52 \pm 0,08^{A}$ & $0,24 \pm 0,25^{\mathrm{B}}$ \\
\hline ALH $(\mu \mathrm{m} / \mathrm{s})$ & $2,76 \pm 0,71^{A}$ & $2,70 \pm 0,12^{A}$ & $2,60 \pm 0,86^{A}$ & $2,57 \pm 0,29^{A}$ & $2,48 \pm 0,77^{A}$ & $1,07 \pm 1,13^{\mathrm{B}}$ \\
\hline $\mathrm{BCF}(\mathrm{Hz})$ & $34,63 \pm 4,35^{\mathrm{A}}$ & $36,58 \pm 1,36^{A}$ & $32,56 \pm 10,71^{A}$ & $35,94 \pm 3,47^{A}$ & $33,43 \pm 8,81^{\mathrm{A}}$ & $18,02 \pm 18,76^{B}$ \\
\hline
\end{tabular}

${ }^{A B C D}$ Letras diferentes correspondem a diferença estatística entre as concentrações em um mesmo parâmetro de motilidade $(p<0,05)$. PH: Extrato hidroalcoólico Polygonum hydropiperoides; MT: Motilidade total, MP: Motilidade progressiva, DAP: Distância média percorrida, DCL: Distância curvilínea, DSL: distância linear, VAP: Velocidade média da trajetória, VCL: Velocidade curvilínea, VSL: Velocidade linear progressiva, BCF: Frequência do batimento flagelar cruzado, ALH: Amplitude do deslocamento lateral da cabeça, STR: Retilinearidade. 
Através da análise por citometria de fluxo, observou-se que o extrato hidroalcoólico de $P$. hydropiperoides nas concentrações de 1,5 até $12,5 \mathrm{mg} \cdot \mathrm{mL}^{-1}$, não causou alterações nos parâmetros avaliados nas células espermáticas quando comparados ao controle $(p>0,05)$ (Tabela 3).

Tabela 3 - Resultado da citometria de fluxo das células espermáticas de suínos (médiađerro padrão) frente às diferentes concentrações do extrato hidroalcoólico de Polygonum hydropiperoides (PH).

\begin{tabular}{|c|c|c|c|c|}
\hline \multirow{2}{*}{ Avaliação } & \multicolumn{4}{|c|}{ Concentração PH (mg.mL $\mathrm{mL}^{-1}$ ) } \\
\hline & 0 (BTS) & 1,5 & 6,2 & 12,5 \\
\hline AR (\%) & $79,61 \pm 1,24^{A}$ & $73,52 \pm 6,54^{A}$ & $75,43 \pm 2,93^{\mathrm{A}}$ & $77,80 \pm 2,19^{\mathrm{A}}$ \\
\hline IM (\%) & $79,91 \pm 2,72^{A}$ & $80,47 \pm 2,18^{A}$ & $78,30 \pm 2,99^{A}$ & $77,60 \pm 3,39^{A}$ \\
\hline DFI & $5,59 \pm 0,01^{A}$ & $5,18 \pm 0,18^{A}$ & $5,72 \pm 0,04^{A}$ & $5,78 \pm 0,02^{A}$ \\
\hline$F(\%)$ & $41,42 \pm 11,71^{A}$ & $18,80 \pm 5,28^{A}$ & $26,30 \pm 3,72^{A}$ & $31,26 \pm 3,42^{A}$ \\
\hline ROS & $26,55 \pm 7,82^{A}$ & $49,44 \pm 13,30^{\mathrm{A}}$ & $43,35 \pm 1,59^{A}$ & $51,91 \pm 2,07^{\mathrm{A}}$ \\
\hline PMM (\%) & $54,20 \pm 10,89^{A}$ & $45,88 \pm 13,17^{A}$ & $49,10 \pm 14,18^{A}$ & $41,23 \pm 16,77^{\mathrm{A}}$ \\
\hline LPO (\%) & $74,91 \pm 3,67^{A}$ & $75,15 \pm 1,28^{A}$ & $75,70 \pm 2,02^{A}$ & $76,91 \pm 5,52^{A}$ \\
\hline
\end{tabular}

Os compostos fenólicos são substâncias que possuem hidroxilas e anéis aromáticos em sua estrutura, provenientes do metabolismo secundário dos vegetais, podendo resultar em uma ação antioxidante ou pró-oxidante, na dependência da estrutura, dose, via de administração ou associação com outros elementos químicos (ANGELO; JORGE, 2007; BIANCHI; ANTUNES, 1999; LIMA et al., 2001).

Alguns dos compostos fenólicos identificados no extrato já têm sido relatados com atividade sobre células espermáticas. Anderson et al. (1997) demonstraram efeito antigenotóxico de miricetina e quercetina em linfócitos e espermatozóides humanos, quando combinadas com mutagênicos alimentares. Purdy et al. (2004) avaliando catequina (25-100 $\mu \mathrm{M})$ na motilidade espermática, relataram efeitos benéficos em todas concentrações testadas quando 
comparados ao controle negativo. Em relação à motilidade progressiva e vigor, a quercetina (5-20 $\mu \mathrm{g} \cdot \mathrm{mL}^{-1}$ ) adicionada em espermatozoides de carneiros, antes da criopreservação, não causou efeitos significativos nos parâmetros (SILVA et al., 2012). Entretanto, Zribi et al. (2012) ao avaliarem a quercetina juntamente com meio de criopreservação em sêmen humano, observaram efeitos benéficos ao descongelamento na motilidade e viabilidade espermática, e integridade de DNA. Entretanto, recentemente, Jamalan et al. (2016) demonstraram que o uso de catequina e quercetina em espermatozóides expostos a metais tóxicos ocasionaram uma diminuição mais intensa da motilidade espermática quando comparado ao controle. Porém, em nosso estudo pode-se observar diferença nos parâmetros de motilidade, provavelmente decorrente da concentração utilizada e dos outros compostos presentes no extrato.

Alterações relacionadas com o tempo, nas 24 e 48 horas (CASA), podem ser provenientes de uma maior produção de ROS pelas mitocôndrias dos espermatozoides, observado na citometria, já que estes necessitam de um maior consumo de oxigênio, por tratarem-se de células com alta motilidade $(>70 \%)$, sendo uma pequena parte deste convertido em ROS (ANDRADE et al., 2010). Além disso, as alterações espermáticas podem ser causadas pelo tempo de contato com os compostos químicos presentes nos extratos e suas concentrações, danificando ou sobrecarregando a célula e/ou disparando algum mecanismo intrínseco, como por exemplo, a reação do acrossoma, além da queda na quantidade de espermatozoides móveis que é considerada proporcional ao tempo de refrigeração (BUCAK; TEKIN, 2007).

Parte das alterações dos parâmetros de motilidade espermática podem estar associadas pela mudança no $\mathrm{pH}$ do meio e adaptação das células espermáticas, já que o extrato de $P$. hydropiperoides, apresentava pH ácido (4,59), o que está de acordo com estudo que descreve queda nos parâmetros de motilidade em $\mathrm{pH}<6,5$ e redução extrema em valores $<5,0$ (CONTRI et al., 2013; ST-PIERRE; MOREAU, 1987). Em determinados momentos, as células regulam seu pH utilizando mecanismos para equilibrar a produção, eliminação, transporte e tamponamento de $\mathrm{H}^{+}$(NISHIGAKI et al., 2014). Sabe-se que em pH mais ácidos $(5,2$ e 6,2$)$ ocorre uma redução na atividade da $\mathrm{Na}^{+} / \mathrm{K}^{+}$-ATPase, a qual participa do equilíbrio 
intracelular de $\mathrm{Ca}_{2}{ }^{+}$, levando a alterações no influxo de cálcio e, consequentemente, a alterações no balanço flagelar, velocidade de natação, capacitação espermática e reação do acrossoma, e na motilidade espermática que depende de concentrações de $\mathrm{Ca}_{2}{ }^{+}$livre (ZHOU et al., 2015). Nas concentrações de 50 e $100 \mathrm{mg} \cdot \mathrm{mL}^{-1}$, houve redução exacerbada na motilidade, sugerindo alta mortalidade dos espermatozoides, o que também acarretaria na produção de ROS, como principal fonte, pela ativação de um sistema enzimático de aminoácidos oxidase (UPRETI et al., 1998). Estudos já relataram que a redução da motilidade espermática in vitro pode ser proveniente de substâncias contidas nos extratos vegetais brutos, entre elas, os polifenóis, flavonoides e ácidos fenólicos (SHEHAB; ABU-GHARBIEH, 2014; TAITZOGLOU et al., 2001). De acordo com alguns autores, os flavonoides, como a catequina, por exemplo, alteram a cascata de fosforilação, interferindo na regulação, sinalização, capacitação e reação acrossomal (PARRISH et al., 1999; PURDY et al., 2004; VISCONTI; KOPF, 1998).

Na avaliação das células espermáticas por citometria de fluxo, pode-se observar um aumento dos níveis de ROS e na fluidez, mesmo não havendo diferença estatística, diferentemente do que foi observado por outros autores, que relataram atividade antioxidante, redução da lipoperoxidação e de danos ao DNA com a utilização de extratos contendo o composto ácido quínico ou quando este foi usado de forma isolada (ALLAl et al., 2016; SENGH et al., 2000, 2005), resultados semelhantes também observados com a quercetina (MCNIVEN; RICHARDSON, 2003, 2006).

A membrana do espermatozóide tem em sua composição grandes quantidades de ácidos graxos insaturados, e estes são os mais atingidos pelos ROS, levando a alterações estruturais e de permeabilidade, que podem provocar a morte celular ou alterar a fluidez, motilidade e viabilidade espermática (AHLUWALIA; HOLMAN, 1969; AM-IN et al., 2011; MAIA; BICUDO, 2009; MILLER et al., 2004). Isso demonstra a importância da diminuição/equilíbrio de ROS, já que estes danificam moléculas de DNA, proteínas e lipídios, e também provocam um esgotamento do ATP, necessário para sua motilidade (DE LAMIRANDE et al., 1998; DE LAMIRANDE; GAGNON, 1992). O aumento da fluidez, em processos fisiológicos, é essencial para capacitação espermática ou alteração da membrana, porém, do ponto de vista tóxico, 
tem sido sugerido como responsável pela diminuição da motilidade espermática devido ao enfraquecimento das ligações de Van der Waals presentes entre as regiões hidrofóbicas dos lipídios, ou pode acarretar em alterações de permeabilidade, permitindo a entrada de íons e pequenas moléculas, vindo a desestabilizar a membrana e provocar danos, levando até mesmo a morte celular (BORGES et al., 2011; SERGENT et al., 2005; SIERRA-VALDEZ et al., 2013).

As alterações provocadas pelo extrato podem sugerir os possíveis mecanismos de ação a serem explorados para aplicação clínica, sendo elucidados através da continuidade dos estudos.

\section{CONCLUSÃO}

O trabalho é pioneiro na avaliação dos efeitos do extrato hidroalcoólico de $P$. hydropiperoides em células espermáticas. O extrato, com composição majoritária de ácido quínico e quercetina, ocasionou alterações somente na motilidade espermática, observadas a partir de $12 \mathrm{mg} \cdot \mathrm{mL}^{-1}$.

\section{EFFECT OF HYDROALCOLIC EXTRACT OF Polygonum hydropiperoides ON SPERMATIC CELLS OF SUs scrofa domesticus}

\section{ABSTRACT}

$P$ olygonum hydropiperoides is one of the plants used as medicine and has biological activities confirmed depending on the chemical composition. In this way, studies on the toxicity and interaction of this plant with the cells are necessary, being spermatozoa innovative options for the evaluation. Thus, the objective of the study was to determine the chemical composition of the hydroacoholic extract of $P$. hydropiperoides $(\mathrm{PH})$ and the toxicity in sperm cells. For the determination of the chemical composition of the extract, the ultra-high performance liquid chromatographic analysis was used. Toxicity was assessed through computerized analysis of sperm motility of domestic swine semen, while cellular changes were analyzed by flow cytometry. A total of 17 phenolic compounds were identified in the plant extract, highlighting quinic acid and quercetin. The parameters evaluated did not change significantly from 1.5 to $6.2 \mathrm{mg} \cdot \mathrm{mL}^{-1}$, regardless the time of exposure to the extract, and the reduction of the parameters occurred progressively when increased the concentrations (6.2 mg. mL ${ }^{-1} \leq 12 \mathrm{mg} \cdot \mathrm{mL}^{-1} \leq 50 \mathrm{mg} \cdot \mathrm{mL}^{-1} \leq 100 \mathrm{mg} \cdot \mathrm{mL}^{-1}$ ). Through flow cytometry no significant changes were observed at the cellular structure level. The alterations caused by the extract may suggest the possible mechanisms of action to be 
explored for clinical application. The hydroalcoholic extract of $P$. hydropiperoides, rich in quinic acid and quercetin, only resulted in changes in sperm motility, from $12 \mathrm{mg} \cdot \mathrm{mL}^{-1}$ on.

Keywords: Toxicity. Plants. Sperm motility. Flow cytometry. CASA.

\section{EFECTO DEL EXTRACTO HIDROALCOHÓLICO DE Polygonum hydropiperoides EN CÉLULAS ESPERMÁTICAS DE SUs scrofa domesticus}

\section{RESUMEN}

$P$ olygonum hydropiperoides es una de las plantas utilizadas como medicinal y que presenta actividades biológicas confirmadas en la dependencia de la composición química. De esta forma, estudios sobre la toxicidad e interacción de esta planta con las células son necesarios, siendo los espermatozoides una opción innovadora para la evaluación. Así, el objetivo del estudio fue determinar la composición química del extracto hidroacohólico de $P$. hydropiperoides $(\mathrm{PH})$ y la toxicidad en células espermáticas. Para la determinación de la composición química del extracto se utilizó el análisis cromatográfico líquido de ultra eficiencia. La toxicidad fue evaluada a través del análisis computarizado de la motilidad espermática de semen porcino doméstico (Sus scrofa domesticus), mientras que las alteraciones celulares fueron analizadas por citometría de flujo. Se identificaron en el extracto vegetal un total de 17 compuestos fenólicos destacándose el ácido quínico y la quercetina. Los parámetros evaluados no sufrieron cambios significativos en las concentraciones de 1,5 a 6,2 mg.mL- ${ }^{1}$ independientemente del tiempo de exposición al extracto, siendo que la reducción de los parámetros ocurrió de forma progresiva al aumento de las concentraciones $\left(6,2 \mathrm{mg} \cdot \mathrm{mL}^{-}{ }^{1} \leq 12 \mathrm{mg} \cdot \mathrm{mL}^{-1} \leq 50 \mathrm{mg} \cdot \mathrm{mL}^{-1} \leq 100 \mathrm{mg} \cdot \mathrm{mL}^{1}{ }^{1}\right.$ ). A través de la citometría de flujo se puedo observar que a nivel de estructura celular, no se observaron cambios significativos. Las alteraciones provocadas por el extracto pueden sugerir los posibles mecanismos de acción a ser explorados para aplicación clínica. El extracto hidroalcohólico de $P$. hydropiperoides, abundante en ácido quínico y quercetina, acarreó sólo cambios en la motilidad espermática a partir de $12 \mathrm{mg} \cdot \mathrm{mL}^{-}{ }^{1}$.

Palabras clave: Toxicidad. Plantas. Motilidad espermática. Citometría de flujo. CASA.

\section{REFERÊNCIAS}

ANGELO, P. M.; JORGE, N. Compostos fenólicos em alimentos - uma breve revisão. Revista do Instituto Adolfo Lutz, v. 66, n. 1, p. 1-9, 2007.

AHLUWALIA, B.; HOLMAN, R. T. Fatty acid composition of lipids of bull, boar, rabbit and human semen. Journal of Reproduction and Fertility, v. 18, n. 3, p. 431-437, 1969. 
ALLAI, L.; DRUART, X.; ÖZTÜRK, M.; et al. Protective effects of Opuntia ficus-indica extract on ram sperm quality, lipid peroxidation and DNA fragmentation during liquid storage. Animal Reproduction Science, v. 175, p. 1-9, 2016.

AM-IN, N.; KIRKWOODB, R. N.; TECHAKUMPHUA, M.; et al. Lipid profiles of sperm and seminal plasma from boars having normal or low sperm motility. Theriogenology, v. 75, n. 5 , p. 897-903, 2011.

ANDERSON, D.; BASARAN, N.; DOBRZYŃSKA, M. M.; et al. Modulating effects of flavonoids on food mutagens in human blood and sperm samples in the comet assay. Teratogenesis, Carcinogenesis, and Mutagenesis, v. 17, n. 2, p. 45-58, 1997.

ANDERSSON, M. C.; MIKKOLA, R.; KOTIAHO, M.; et al. Boar spermatozoa as a tool in toxicological research, a novel application for extended boar semen commercially produced by Al stations. Reproduction in Domestic Animals, v. 41, n. 4, p. 303-303, 2006.

ANDERSSON, M. A.; MIKKOLA, R.; RASIMUS, S.; et al. Boar spermatozoa as a biosensor for detecting toxic substances in indoor dust and aerosol. Toxicology in Vitro, v. 24, n. 7, p. 2041-2052, 2010.

ANDRADE, E. R.; MELO-STERZA, F. A.; SENEDA, M. M.; et al. Consequências da produção das espécies reativas de oxigênio na reprodução e principais mecanismos antioxidantes. Revista Brasileira de Reprodução Animal, v. 34, n. 2, p. 79-85, 2010.

BARBOSA-FILHO, J. M.; NASCIMENTO JÚNIOR, F. A.; TOMAZ, A. C. A.; et al. Natural products with antileprotic activity. Revista Brasileira de Farmacognosia, v. 17, n. 1, p. 141-148, 2007.

BIANCHI, M. L. P.; ANTUNES, L. M. G. Radicais livres e os principais antioxidantes da dieta. Revista de Nutrição, v. 12, n. 2, p. 123-130, 1999.

BIANCHI, N. R.; SILVA, M. O.; SPIASSI, L.; et al. Ensaio de toxicidade excessiva e "screening" fitoquímico de plantas usadas popularmente no tratamento de afecções gastro-intestinais. Revista Brasileira de Farmácia, v. 74, p. 50-51, 1993.

BORGES, C.; SILVA, M. R.; GUIMARÃES, J. D.; et al. Membrana plasmática de espermatozoides bovinos: efeito de metabólitos do oxigênio, antioxidantes e criopreservação. Revista Brasileira de Reprodução Animal, v. 35, n. 3, p. 303-314, 2011.

BRANDÃO, M.; LACA-BUENDIA, J. P.; GAVILANES, M. L. Plantas palustres e aquáticas que se comportam como invasoras, no estado de Minas Gerais. Acta Botanica Brasilica, v. 2, n. 1, p. 255-265, 2012.

BRASIL. Ministério da Saúde elabora Relação de Plantas Medicinais de Interesse ao SUS, 2009a. Disponível em: 
<http://bvsms.saude.gov.br/bvs/sus/pdf/marco/ms_relacao_plantas_medicinais_sus_0603. pdf $>$. Acesso em: 21 jan. 2017.

BRASIL. Programa Nacional de Plantas Medicinais e Fitoterápicos/Ministério da Saúde, Secretaria de Ciência, Tecnologia e Insumos Estratégicos, Departamento de Assistência Farmacêutica e Insumos Estratégicos, 2009b. Disponível em:

<http://bvsms.saude.gov.br/bvs/publicacoes/programa_nacional_plantas_medicinais_fitote rapicos.pdf>. Acesso em: 01 dez. 2016.

BUCAK, M. N.; TEKIN, N. Protective effect of taurine, glutathione and trehalose on the liquid storage of ram semen. Small Ruminant Research, v. 73, n. 1-3, p. 103-108, 2007.

BUSSMANN, R. W.; MALCA-GARCÍA, G.; GLENN, A.; et al. Minimum inhibitory concentrations of medicinal plants used in Northern Peru as antibacterial remedies. Journal of Ethnopharmacology, v. 132, n. 1, p. 101-108, 2010.

BUSSMANN, R. W.; SHARON, D.; PEREZ, F. A.; et al. Antibacterial activity of northernperuvian medicinal plants. Arnaldoa, v. 15, n. 1, p. 127-148, 2008.

CECHINEL FILHO, V.; YUNES, R. A. Estratégias para a obtenção de compostos farmacologicamente ativos a partir de plantas medicinais. Conceitos sobre modificação estrutural para otimização da atividade. Química Nova, v. 21, n. 1, p. 99-105, 1998.

CONTRI, A.; GLORIA, A.; ROBBE, D.; et al. Kinematic study on the effect of pH on bull sperm function. Animal Reproduction Science, v. 136, n. 4, p. 252-259, 2013.

CUNHA, F. A. B.; WACZUK, E. P.; DUARTE, A. E.; et al. Cytotoxic and antioxidative potentials of ethanolic extract of Eugenia uniflora L. (Myrtaceae) leaves on human blood cells.

Biomedicine \& Pharmacotherapy, v. 84, p. 614-621, 2016.

DE LAMIRANDE, E.; GAGNON, C. Reactive oxygen species and human spermatozoa. II. Depletion of adenosine troposphere plays an important role in the inhibition of sperm motility. Journal of Andrology, v. 13, n. 5, p. 379-386, 1992.

DE LAMIRANDE, E.; TSAI, C.; HARAKAT, A.; et al. Involvement of reactive oxygen species in human sperm acrosome reaction induced by $A 23187$, lysophosphatidylcholine, and biological fluid ultra filtrates. Journal of Andrology, v. 19, n. 5, p. 585-594, 1998.

DOMÍNGUEZ-REBOLLEDO, A. E.; MARTíNEZ-PASTOR, F.; BISBAL, A. F.; et al. Response of thawed epididymal red deer spermatozoa to increasing concentrations of hydrogenperoxide, and importance of individual male variability. Reproduction in Domestic Animals, v. 46, n. 3, p. 393-403, 2010.

EVENSON, D.; JOST, L. Sperm chromatin structure assay: DNA denaturability. Methods in Cell Biology, v. 42, p. 159-176, 1994. 
FERNANDEZ-GAGO, R.; DOMINGUEZ, J. C.; MARTINEZ-PASTOR, F. Seminal plasma applied post-thawing affects boar sperm physiology: a flow cytometry study. Theriogenology, v. 80, n. 4, p. 400-410, 2013.

FREIXA, B.; VILA, R.; VARGAS, L.; et al. Screening for antifungal activity of nineteen latin american plants. Phytotherapy research, v. 12, n. 6, p. 427-430, 1998.

HOORNSTRA, D.; ANDERSSON, M. A.; MIKKOLA, R.; et al. A new method for in vitro detection of microbially produced mitochondrial toxins. Toxicology in Vitro, v. 17, n. 5-6, p. 745-751, 2003.

JÄÄSKELÄINEN, E. L.; TEPLOVA, V.; ANDERSSON, M. A.; et al. In vitro assay for human toxicity of cereulide, the emetic toxin produced by food poisoning Bacillus cereus. Toxicology in Vitro, v. 17, n. 5-6, p. 737-744, 2003.

JAMALAN, M.; GHAFFARI, M. A.; HOSEINZADEH, P.; et al. Human sperm quality and metal toxicants: Protective effects of some flavonoids on male reproductive function. International Journal of Fertility and Sterility, v. 10, n. 2, p. 215-222, 2016.

LIMA, L. R. P.; OLIVEIRA, T. T.; NAGEM, T. J.; et al. Bixina, Norbixina e Quercetina e seus efeitos no metabolismo lipídico de coelhos. Brazilian Journal of Veterinary Research and Animal Science, v. 38, n. 4, p. 196-200, 2001.

LORENZI, H.; MATOS, F. J. A. Plantas medicinais no Brasil: nativas e exóticas. 2. ed. Nova Odessa: Plantarum, 2008. 512p.

MAIA, M. S.; BICUDO, S. D. Radicais livres, antioxidantes e função espermática em mamíferos: uma revisão. Revista Brasileira de Reprodução Animal, v. 33, n. 4, p. 183-193, 2009.

MARTINEZ-ALBORCIA, M. J.; MORRELL, J. M.; GIL, M. A.; et al. Suitability and effectiveness of single layer centrifugation using Androcoll-P in the cryopreservation protocol for boar spermatozoa. Animal Reproduction Science, v. 140, n. 3-4, p. 173-179, 2012.

MARTINEZ-PASTOR, F.; MARTÍNEZ, F.; GARCÍA-MACÍAS, V.; et al. A pilot study on postthawing quality of Iberian red deer spermatozoa (epididymal and electroejaculated) depending on glycerol concentration and extender osmolality. Theriogenology, v. 66, n. 5, p. 1165-1172, 2010.

MCNIVEN, M. A.; RICHARDSON, G. F. Chilled storage of stallion semen using perfluorochemicals and antioxidants. Cell Preservation Techonology, v. 1, n. 3, p. 165-174, 2003. 
MCNIVEN, M. A.; RICHARDSON, G. F. Effect of Quercetin on Capacitation Status and Lipid Peroxidation of Stallion Spermatozoa. Cell Preservation Technology, v. 4, n. 3, p. 169-177, 2006.

MILLER, R. R.; SHEFFER, C. J.; CORNETT, C. L.; et al. Sperm membrane fatty acid composition in the Eastern grey kangaroo (Macropus giganteus), koala (Phascolarctos cinereus), and common wombat (Vombatus ursinus) and its relationship to cold shock injury and cryopreservation success. Cryobiology, v. 49, n. 2, p. 137-148, 2004.

NISHIGAKI, T.; JOSÉ, O.; GONZÁLEZ-COTA, A. L.; et al. Intracellular pH in sperm physiology. Biochemical and Biophysical Research Communications, v. 450, n. 3, p. 1149-1158, 2014.

PARRISH, J. J.; SUSKO-PARRISH, J. L.; GRAHAM, J. K. In vitro capacitation of bovine spermatozoa: role of intracellular calcium. Theriogenology, v. 51, n. 2, p. 461-472, 1999.

PRASHAR, A.; HILI, P.; VENESS, R. G.; et al. Antimicrobial action of palmarosa oil (Cymbopogon martinii) on Saccharomyces cerevisiae. Phytochemistry, v. 63, n. 5, p. 569-575, 2003.

PURDY, P. H.; ERICSSON, S. A.; DODSON, R. E.; et al. Effects of the flavonoids, silibinin and catechin, on the motility of extended cooled caprine sperm. Small Ruminant Research, v. 55, n. 1-3, p. 239-243, 2004.

SCHIEDECK, G.; BEVILAQUA, G. A. P.; BACHTIGAL, G. F.; et al. Método de preparo de tintura de plantas bioativas para fins agrícolas. Embrapa Clima Temperado, Comunicado Técnico, $\mathrm{n}$. 190, p. 1-4, 2008.

SENGH, Y.; ÅKESSON, C.; HOLMGREN, K.; et al. An active ingredient of Cat's Claw water extracts identification and efficacy of quinic acid. Journal of Ethnopharmacology, v. 96, n. 3, p. 577-584, 2005.

SENGH, Y.; BRYNGELSSON, C.; PERO, R. W. Enhanced DNA repair immune function and reduced toxicity of C-MED100TM, a novel aqueous extract from Uncaria tomentosa. Journal of Ethnopharmacology, v. 69, n. 2, p. 115-126, 2000.

SERGENT, O.; PEREIRA, M.; BELHOMME, C.; et al. Role for membrane fluidity in ethanolinduced oxidative stress of primary rat hepatocytes. Journal of Pharmacology and Experimental Therapeutics, v. 313, n. 1, p. 104-111, 2005.

SEVERIN, I.; DAHBI, L.; LHUGUENOT, J. C.; et al. Safety assessment of food-contact paper and board using a battery of short-term toxicity tests: European Union BIOSAFEPAPER Project. Food Additives \& Contaminants, v. 22, n. 10, p. 1032-1041, 2005.

SHEHAB, N. G.; ABU-GHARBIEH, E. Phenolic Profiling and Evaluation of Contraceptive Effect of the Ethanolic Extract of Salsola imbricata Forssk. In Male Albino Rats. Evidence-Based 
Complementary and Alternative Medicine, v. 2014, p. 1-8, 2014. doi.org/10.1155/2014/695291

SIERRA-VALDEZ, F. J.; FORERO-QUINTERO, L. S.; ZAPATA-MORIN, P. A.; et al. The Influence of Non Polar and Polar Molecules in Mouse Motile Cells Membranes and Pure Lipid Bilayers. PLOS ONE, v. 8, n. 4, p. 1-6, 2013.

SILVA, C. M. B.; ORTEGA FERRUSOLA, C.; MORILLO RODRIGUEZ, A.; et al. Sex sorting increases the permeability of the membrane of stallion spermatozoa. Animal Reproduction Science, v. 138, n. 3-4, p. 241-251, 2013.

SILVA, E. C. B.; CAJUEIRO, J. F. P.; SILVA, S. V.; et al. Effect of antioxidants resveratrol and quercetin on in vitro evaluation of frozen ram sperm. Theriongelonogy, v. 77, n. 8, p. 17221726, 2012.

SILVA, E. F.; VARELA-JUNIOR, A. S.; CARDOSO, T. F.; et al. Reproductive toxicology of 2,4 dinitrophenol in boar sperm. Toxicology in Vitro, v. 35, p. 31-35, 2016.

ST-PIERRE, M.; MOREAU, G. Résistance et viabilité des gamètes d'Omble de fontaine, Salvelinus fontinalis, à différents pH. Hydrobiologia, v. 153, n. 2, p. 139-148, 1987.

TAITZOGLOU, I. A.; TSANTARLIOTOU, M.; ZERVOS, I.; et al. Inhibition of human and ovine acrosomal enzymes by tannic acid in vitro. Reproduction, v. 121, n. 1, p. 131-137, 2001.

UPRETI, G. C.; JENSEN, K.; MUNDAY, R.; et al. Studies on aromatic amino acid oxidase activity in ram spermatozoa: role of pyruvate as an antioxidant. Animal Reproduction Science, v. 51, n. 4, p. 275-287, 1998.

VISCONTI, P. E.; KOPF, G. S. Regulation of protein phosphorylation during sperm capacitation. Biology of Reproduction, v. 59, n. 1, p. 1-6, 1998.

ZHOU, J.; CHEN, L.; LI, J. et al. The Semen pH Affects Sperm Motility and Capacitation. PLOS ONE, v. 10, n. 7, p. 1-15, 2015.

ZRIBI, N.; CHAKROUN, N. F.; ABDALLAH, F. B.; et al. Effect of freezing-thawing process and quercetin on human sperm survival and DNA integrity. Cryobiology, v. 65, n. 3, p. 326-331, 2012.

ZÖLLNER, T.; SCHWARZ, M. Herbal Reference Standards: applications, definitions and regulatory requirements. Revista Brasileira de Farmacognosia, v. 23, n. 1, p. 1-21, 2013.

Autor para correspondência: Claudia Giordani. Universidade Federal de Pelotas, Faculdade de Veterinária, Campus Universitário S/N, Capão do Leão (RS), CEP 96010-900, Brasil. claarte@hotmail.com 